\title{
An Adaptation System to Avoid Apoptosis via Autophagy Under Hypoxic Conditions in Pancreatic Cancer Cells
}

\author{
SATOSHI OWADA ${ }^{1 *}$, KANAKO ITO $^{1 *}$, HITOSHI ENDO $^{1}$, YUKARI SHIDA $^{1}$, \\ CHISA OKADA ${ }^{2}$, TAKAHIRO NEZU ${ }^{1}$ and MASAYUKI TATEMICHI ${ }^{1}$ \\ ${ }^{1}$ Center for Molecular Prevention and Environmental Medicine, \\ Department of Preventive Medicine, Tokai University School of Medicine, Isehara, Japan; \\ ${ }^{2}$ Support Center for Medical Research and Education, Tokai University, Isehara, Japan
}

\begin{abstract}
Background/Aim: Pancreatic cancer tissue is a hypoxic environment resistant to anticancer drugs. This study examined the role of autophagy as a response to hypoxic stress in pancreatic cancer. Materials and Methods: Pancreatic cell lines (PANC-1, BxPC-3 and AsPC-1) were exposed to hypoxic conditions using cobalt chloride, a hypoxia-mimicking agent. Protein expression and cytotoxicity assays were performed to determine the effect of hypoxia on autophagy. Results: When pancreatic cancer cells were exposed to hypoxia, autophagy was induced. The autophagy-inducing signal was dependent on the AMPK pathway. Inhibition of autophagy in a hypoxic state induced a remarkable cytotoxicity and enhanced apoptosis. When an AMPK inhibitor was added, cytotoxicity was observed in the hypoxic environment. Conclusion: The induced autophagy, dependent on the AMPK pathway, is a necessary survival strategy adopted by pancreatic cancer cells to adapt to hypoxic stress, and could be an attractive target for drug development.
\end{abstract}

Pancreatic cancer has a high mortality rate with a high malignancy grade, a 5-year survival rate of $<5 \%$, and a median survival of 4-6 months in Japan, similar to other countries $(1,2)$. Pancreatic cancer is treated with gemcitabine or TS-1 as standard therapy, but clinical outcomes are unfortunately unfavorable (3).

*These Authors contributed equally to this study.

Correspondence to: Dr. Masayuki Tatemichi, Center for Molecular Prevention and Environmental Medicine, Department of Preventive Medicine, Tokai University School of Medicine, Isehara, Japan. Tel: +81463931121 (ext. 2611), e-mail: tatemichi@tokai.ac.jp

Key Words: Pancreatic cancer, hypoxia, AMPK, autophagy, cytotoxicity.
Solid tumors contain premature vascular networks as a result of disorganized growth of cancer cells and imbalanced angiogenesis (4-7). Pancreatic cancer tissue, in particular, is known to be hypovascularized. Since nutrients and oxygen are supplied to cancer tissues via the bloodstream, the cancer microenvironment is exposed to a hypoxic, low-nutrient environment. Many reports have described a significant reduction of the effect of anticancer drugs in the cancer microenvironment (8-11), and thus a new target for drug development is required.

Stabilization of the transcription factor hypoxia inducible factor-1 (HIF-1) plays an important role in the mechanism that allows cells to adapt to a hypoxic environment (12), a characteristic of cancer tissue. HIF-1 has more than 800 downstream target genes, and regulates their expression for adaptation to hypoxic conditions. It was recently reported that induction of autophagy is dependent on HIF-1 and supports cell survival (13). Autophagy is a degradation mechanism that occurs in lysosomes and is known to have two functions. One function is to degrade cytoplasmic components within the cell via lysosomes, and thereby generate degradation products such as amino acids. Under normal conditions, these activities mainly function as an intracellular recycling system. The other function of autophagy is intracellular quality control or disposal of intracellular waste products. These functions are induced when the cell must appropriately dispose of damaged organelles or proteins. Autophagy is induced when cells are under large stresses, such as metabolic stresses including hypoxia or nutrient deprivation, or administration of anticancer drugs (14-17). Cells can take advantage of the autophagy system to recycle organelles or proteins damaged under hostile environments. Thus, autophagy enables adaptation to a hypoxic environment. However, the roles of autophagy induced by hypoxic environments vary depending on primary organs and cell types, and no conclusion has been drawn regarding whether autophagy provides advantages or disadvantages in terms of cell survival (18-20). 
The significance of autophagy in each cancer or cell type, or its potential as a therapeutic target, requires investigation. This study aimed to determine the significance of autophagy in pancreatic cancer cell lines under hypoxic conditions as well as the potential of autophagy as a therapeutic target. We have shown that under a hypoxic environment, apoptosis is avoided through induction of autophagy dependent on the AMPK pathway. Inhibition of this pathway could be a new target of drug development for hypoxic adaptation of cells in pancreatic cancer.

\section{Materials and Methods}

Reagents. Materials were obtained from the following sources: LC3 antibody from MBL (Nagoya, Japan); phospho-p70 S6 kinase (Thr389), p70 S6 kinase antibody, phospho-Akt (Ser473), AKT antibody, phospho-AMPK $\alpha$ (Thr172) antibody, AMPK $\alpha$ antibody, Alexa Fluor 555 conjugate, and Alexa Fluor 488 conjugate from Cell Signaling Technologies (Beverly, MA, USA); actin antibody from Wako Pure Chemical Industries (Osaka, Japan); LAMP1 antibody from Santa Cruz Biotechnology (Santa Cruz, CA, USA); compound C and LY294002 from Calbiochem-Merck (Darmstadt, Germany); Dulbecco's Modified Eagle's medium (DMEM), dimethylsulfoxide (DMSO), cobalt chloride, bafilomycin A1, and a proteinase inhibitor cocktail from Sigma (St. Louis, MO, USA); and HIF-1 $\alpha$ antibody from GeneTex (Irvine, CA, USA).

Cell lines and culture conditions. The pancreatic cancer cell lines PANC-1, BxPC-3 and AsPC-1 were purchased from ECACC (European Collection of Cell Cultures, Salisbury, UK). All cell lines were cultured in DMEM supplemented with $10 \%$ fetal bovine serum, $50 \mathrm{U} / \mathrm{ml}$ penicillin, $50 \mu \mathrm{g} / \mathrm{ml}$ streptomycin, and non-essential amino acids (Gibco BRL, Paisley, UK).

Cytotoxicity assay. A cytotoxicity assay was performed using Cell Counting kit-8 (Dojindo Molecular Technologies, Kumamoto, Japan). In brief, PANC-1 cells and BxPC-3 cells were seeded in 96well plates $\left(5 \times 10^{3} /\right.$ well $)$ and incubated in fresh DMEM medium for $24 \mathrm{~h}$. The medium was changed to either DMEM with or without $300 \mu \mathrm{M}$ cobalt chloride followed by treatment with serial dilutions of either autophagy inhibitors or compound C. After $24 \mathrm{~h}$ incubation, $90 \mu \mathrm{l}$ of DMEM medium with a $10 \%$ WST- 8 solution was added to the well, and the plate was incubated for a further $4 \mathrm{~h}$. Next, absorbance was measured at $460 \mathrm{~nm}$. The cell number in the absence of autophagy inhibitors or AMPK inhibitor under each culture condition was set as $100 \%$.

Western blot analysis. Protein extraction and Western blot analysis were performed as described previously (21). The antibody dilutions used were in accordance with the manufacturers' instructions.

Immunostaining analysis. Images of optical sections with $0.7-\mu \mathrm{m}$ thickness were captured using a Zeiss LSM700 confocal laser scanning microscope (Carl Zeiss Microscopy, GmbH, Jena, Germany) with the $63 \times$ (N.A. 1.2) objective lens. The Alexa Fluor 488 dye was excited by the $488 \mathrm{~nm}$ laser line, and the resulting fluorescent emission was detected through the filter that transmitted wavelengths ranging from 420 to $550 \mathrm{~nm}$. The Alexa Fluor 555 dye was excited by the $555 \mathrm{~nm}$ laser line, and the resulting fluorescent emission was detected through the filter that transmitted a wavelength of over $560 \mathrm{~nm}$. The number of puncta was counted using Image J v1.51n software (NIH, Bethesda, MD, USA). 4',6diamidino-2-phenylindole dihydrochloride (DAPI) was used for nuclei counterstaining, and the 405-nm-excited fluorescent emission of DAPI was detected through the filter that transmitted a wavelength of over $420 \mathrm{~nm}$.

Statistical analysis. Bars or symbols in the graph represent means \pm SD generated from at least 3 independent experiments. Significant differences were determined by one-way analyses of variance (ANOVA) or $t$-test. A $p$-value $<0.05$ was considered statistically significant.

\section{Results}

Autophagy induced by hypoxic stress. To examine whether autophagy could be induced by a hypoxic environment, cobalt chloride $\left(\mathrm{CoCl}_{2}\right)$, a compound known to mimic hypoxia, was added to cell lines $(22,23)$. The hypoxic marker HIF-1 was significantly increased in pancreatic cancer cells after addition of $\mathrm{CoCl}_{2}$ (Figure 1A). In addition, treatment with $\mathrm{CoCl}_{2}$ enhanced expression of LC3-II, a marker of autophagy, in all 3 cell lines, which suggests that autophagy was induced (Figure 1A). The distribution of LC3 and lysosome-associated membrane protein 1 (LAMP1), a lysosome marker, were observed using cell immunostaining (Figure 1B). Treatment with $\mathrm{CoCl}_{2}$ increased the number of LC3 puncta, indicating that autophagy was induced (Figure $1 \mathrm{~B}$ and C). Furthermore, the distribution of most of the LC3 puncta corresponded to the lysosomes, and the fusion of autophagosomes and lysosomes was also observed (Figure $1 \mathrm{~B}$ and $\mathrm{D})$. These results strongly suggested that autophagy was induced in pancreatic cancer cell lines under hypoxic environments.

Autophagy induced by a hypoxic environment is dependent on the AMPK/mTOR pathway. A detailed analysis of the autophagy signaling pathway induced by a hypoxic environment was performed. The activity of mTOR, a known regulating factor of autophagy, was examined by observing the phosphorylation level of S6 kinase (S6K), a direct downstream target of mTOR (24). Addition of $\mathrm{CoCl}_{2}$ dramatically reduced $\mathrm{S} 6 \mathrm{~K}$ phosphorylation, which suggests decreased mTOR activity (Figure 2A). In addition, phosphorylation of AKT and AMPK, upstream molecules of the mTOR pathway, was increased (Figure 2B). Phosphorylation of mTOR is maintained by activation of AKT or inactivation of AMPK (25). Thus, autophagy induced by a hypoxic environment did not appear to be dependent on AKT, and could have been mediated by a signaling pathway dependent on the AMPK/mTOR pathway.

Cellular damage specific to the hypoxic environment caused by inhibition of autophagy. A CCK8 assay was performed to 
A

\begin{tabular}{|c|c|c|}
\hline \multicolumn{3}{|c|}{$\mathrm{CoCl}_{2} 300 \mu \mathrm{M}$} \\
\hline PANC-1 & AsPC-1 & BxPC-3 \\
\hline
\end{tabular}

LC3 (Short Expo)

LC3 (Long Expo)

HIF-1a

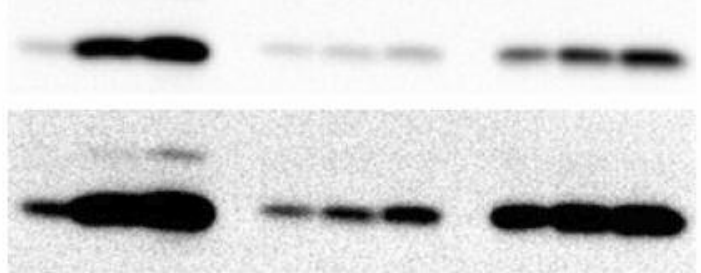

\author{
HF-1 $\alpha$
}

- 56 뿌

ACTIN

B

$\mathrm{CoCl}_{2} \mathrm{O} \mu \mathrm{M}$

$\mathrm{CoCl}_{2} 300 \mu \mathrm{M}$
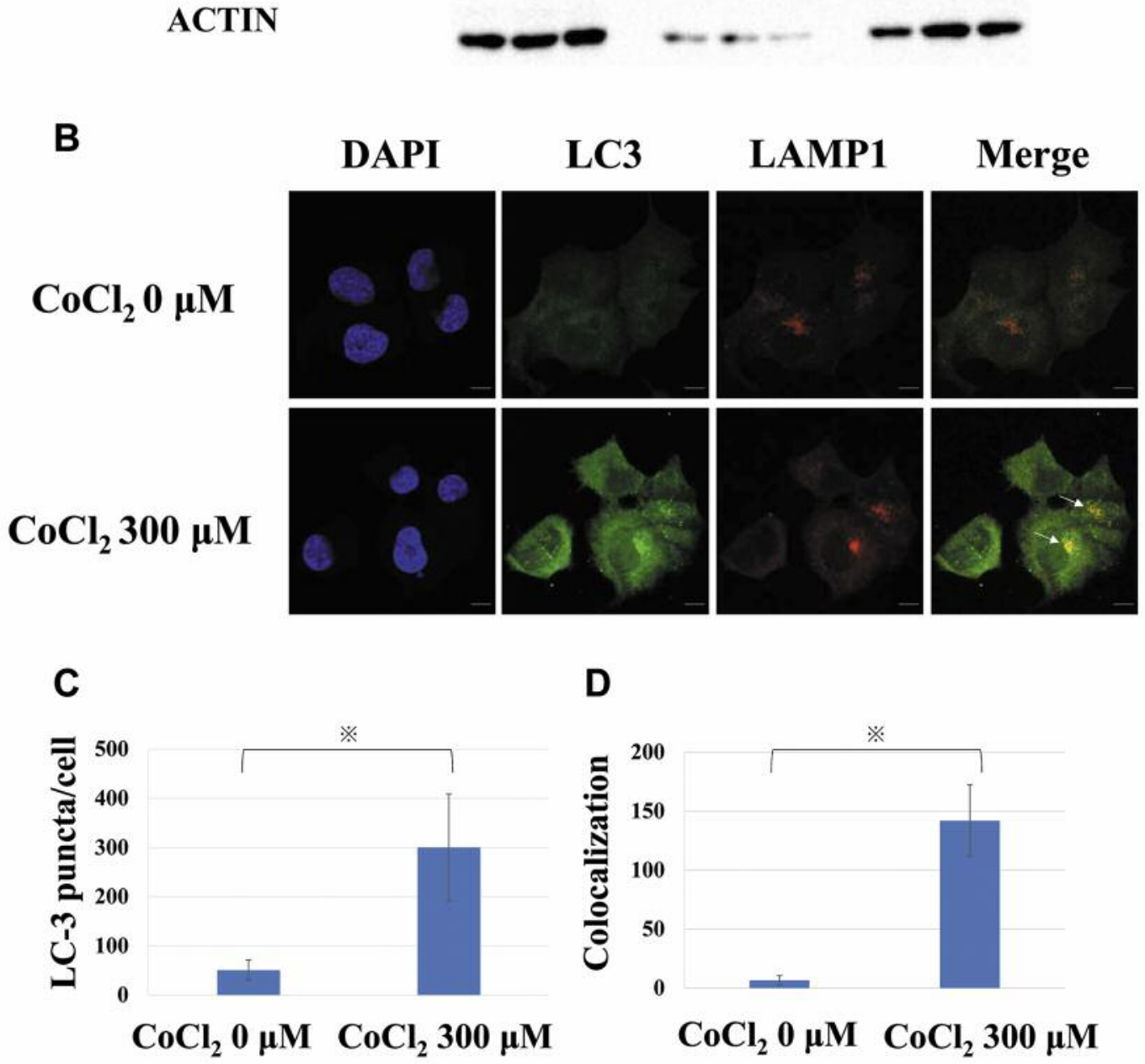

D

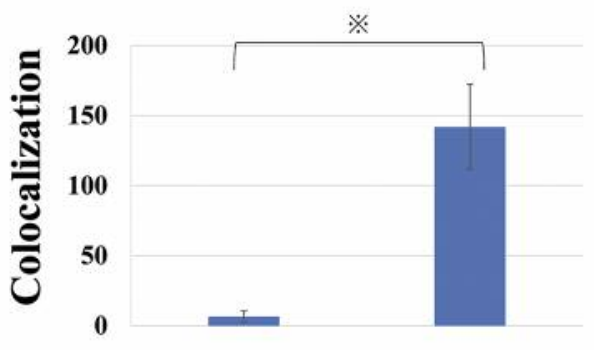

\title{
$\mathrm{CoCl}_{2} \mathrm{O} \mu \mathrm{M} \quad \mathrm{CoCl}_{2} 300 \mu \mathrm{M}$
}

Figure 1. Treatment of cobalt chloride induces progressive activation of autophagy in pancreatic cancer cell lines. PANC-1, AsPC-1 and BxPC-3 cells were treated with $300 \mu \mathrm{M}$ cobalt chloride and harvested at the indicated incubation times. Expression levels of LC3, HIF-1 $\alpha$ and actin were determined by western blot analysis (A). PANC-1 cells were immunostained with anti-LC3 (green) and anti-LAMP1 (lysosome marker, red). Insets indicate magnified images of the boxed area. Bar, $10 \mu \mathrm{m}(B)$. Punctate dots of LC3 in each cell were counted $\left({ }^{*} p<0.05\right)(C)$, and colocalization between $L C-3$ and lysosomes was assessed in each cell using Image $J\left({ }^{*} p<0.05\right)(D)$. 
A

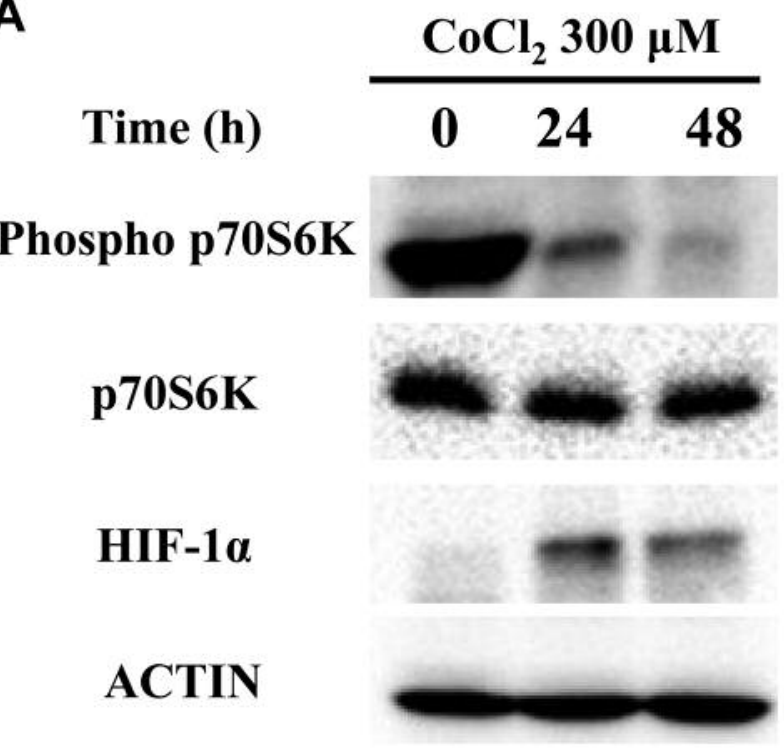

B

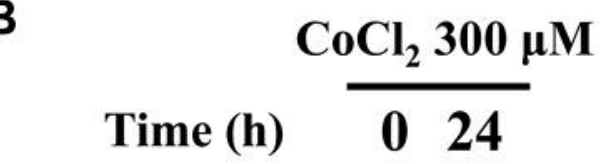

Phospho AMPK

\author{
AMPK \\ Phospho AKT
}

AKT

HIF1a
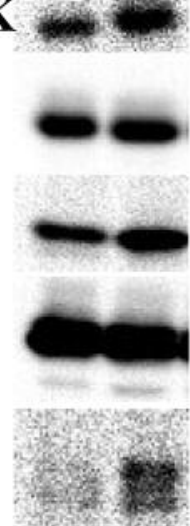

Figure 2. Autophagic flux induced by cobalt chloride treatment was AMPK/mTOR-dependent. PANC-1 cells were treated with $300 \mu M$ cobalt chloride and harvested at the indicated incubation times. Expression levels of phospho-P70S6K, P70S6K, HIF-1 $\alpha$, and actin were determined by western blot analysis (A). Expression levels of phospho-AKT, AKT, phospho-AMPK, AMPK, and HIF-1a were determined by western blot analysis (B).

determine how cell survival is affected by autophagy induced by a hypoxic environment. An autophagy-specific inhibitor, bafilomycin $(26,27)$, was added to pancreatic cancer cell lines. Treatment with bafilomycin showed no cytotoxicity in the cells under normal culture conditions but resulted in significant cytotoxicity under a hypoxic environment (Figure 3A). Treatment with LY294002, an autophagy inhibitor (28), also showed a hypoxic environment-selective cytotoxicity (Figure 3B). The role of AMPK in autophagy induced during the adaptation to hypoxic environments was examined. The use of an AMPK inhibitor, compound C (29), resulted in marked cytotoxicity under a hypoxic environment (Figure $3 \mathrm{C})$. These results reveal that adaptation to hypoxic environments requires a functional autophagy process, and that the AMPK pathway plays a key role in autophagy signaling.

Autophagy induced by hypoxia inhibits apoptosis. Activation of autophagy has been reported to inhibit apoptosis and support cell survival (30). Induction of apoptosis was examined in the presence of an autophagy inhibitor, which blocks the adaptation of cells to hypoxia. Cleavage of poly (ADP-ribose) polymerase (PARP), a marker of apoptosis, was strongly expressed under a hypoxic environment with autophagy-inhibited conditions (Figure 4A). In addition, treatment with z-VAD, an apoptosis inhibitor, resulted in a significant reduction in cell death caused by inhibition of autophagy under a hypoxic environment (Figure 4B). These results demonstrate that pancreatic cancer cell survival and adaptation to hypoxic environments is promoted by apoptosis inhibition mediated by autophagy.

\section{Discussion}

We investigated the importance of autophagy for adaptation to hypoxia, a characteristic of the cancer microenvironment, using pancreatic cancer cell lines reflecting a hypovascular form of cancer. We determined that autophagy induced by a hypoxic environment involves the AMPK pathway and inhibits apoptosis. A significant cytotoxicity resulting from treatment with an autophagy inhibitor suggests that blocking this pathway could be a target for drug development to inhibit pancreatic cancer adaptation to hypoxia.

If the autophagy induction dependent on HIF-1 is stabilized under hypoxic stress, the use of a HIF-1 inhibitor could be an effective treatment method. HIF-1 not only plays a role in autophagy, but also helps cancers become malignant by shifting the source of energy production to the glycolysis system or enhancing angiogenesis (12). However, from the standpoint of the cancer microenvironment, cancer tissues are exposed not only to hypoxia, but also to a nutrient-poor environment, as nutrients are also supplied through the bloodstream. There have been some reports on resistance to anticancer drugs independently of HIF-1, even in lownutrient environments $(31,32)$. Thus, identification of a therapeutic target for the cancer microenvironment requires 


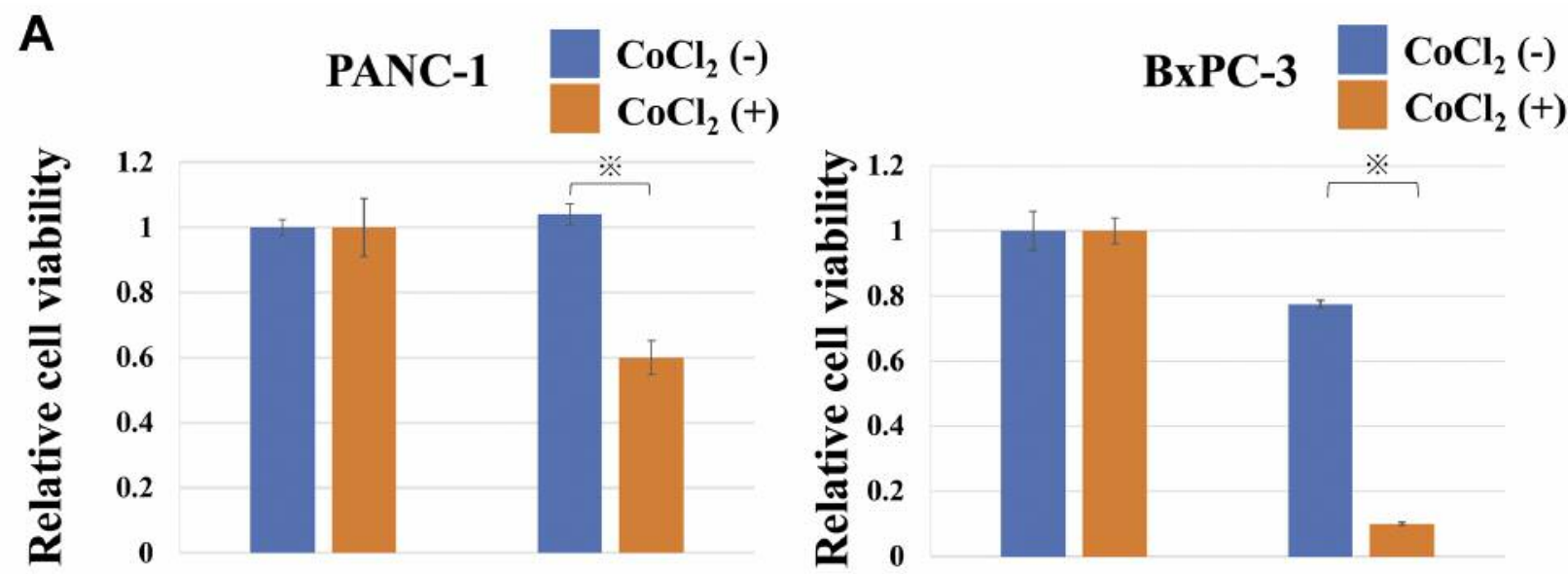

\section{Control Bafilomycin (50 $\mathrm{nM}) \quad$ Control Bafilomycin $(50 \mathrm{nM})$}

B

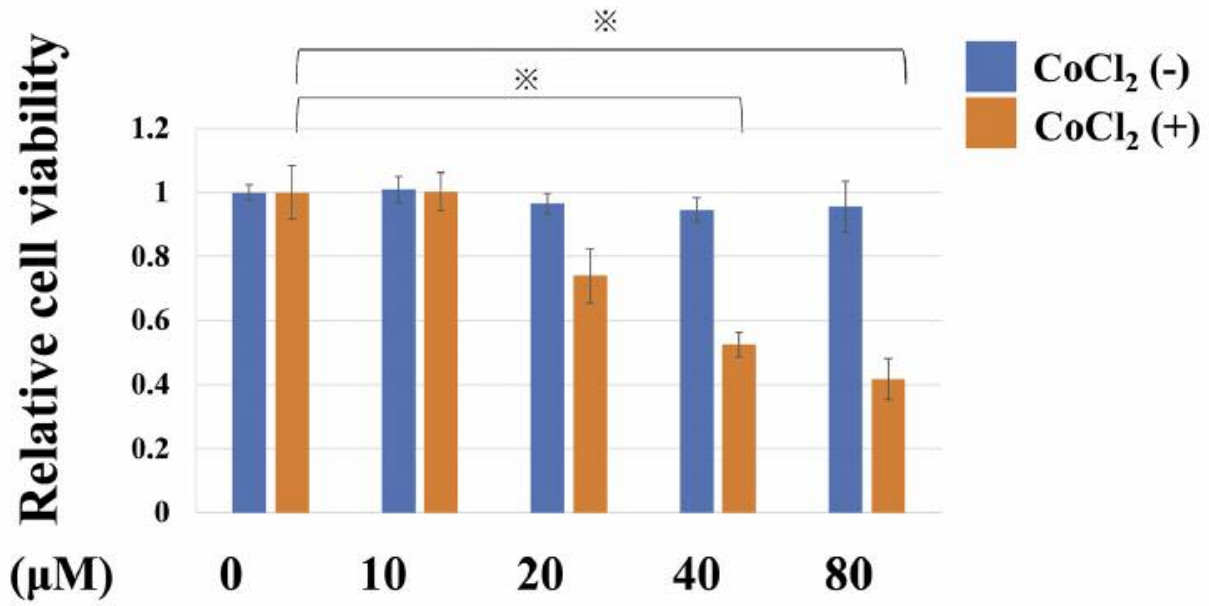

C
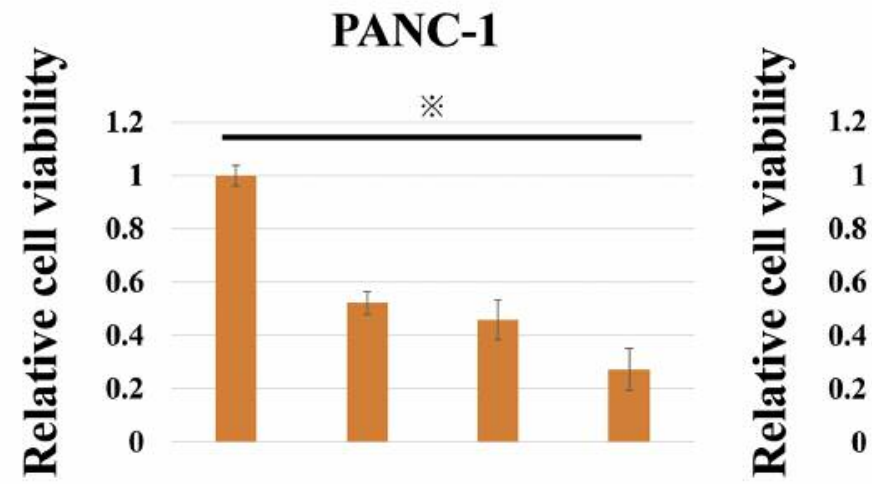

Compound $\mathrm{C}(\mu \mathrm{M}) \quad 0 \quad 5 \quad 10 \quad 20$

0

BxPC-3

Figure 3. Hypoxia-selective cytotoxicity arising from autophagy inhibition. Cell viability was assessed using a CCK8 assay. BxPC-3 and PANC-1 cells were treated with bafilomycin or LY294002 under either standard culture conditions or $300 \mu M$ cobalt chloride treatment for $24 h(* p<0.05)$ (A, B). PANC-1 cells were treated with compound $C$ under $300 \mu M$ cobalt chloride treatment for $24 h\left({ }^{*} p<0.05\right)(C)$. 

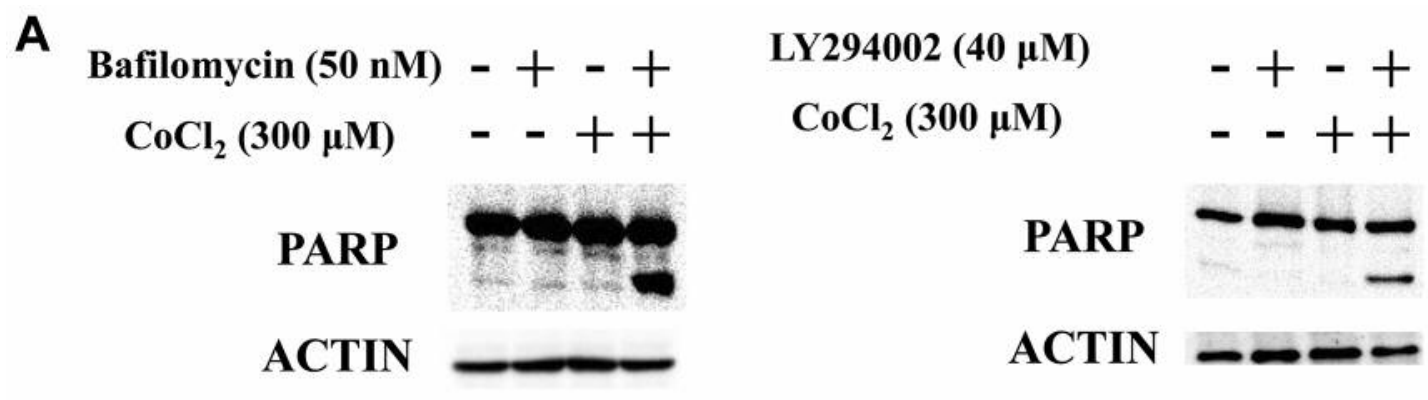

B

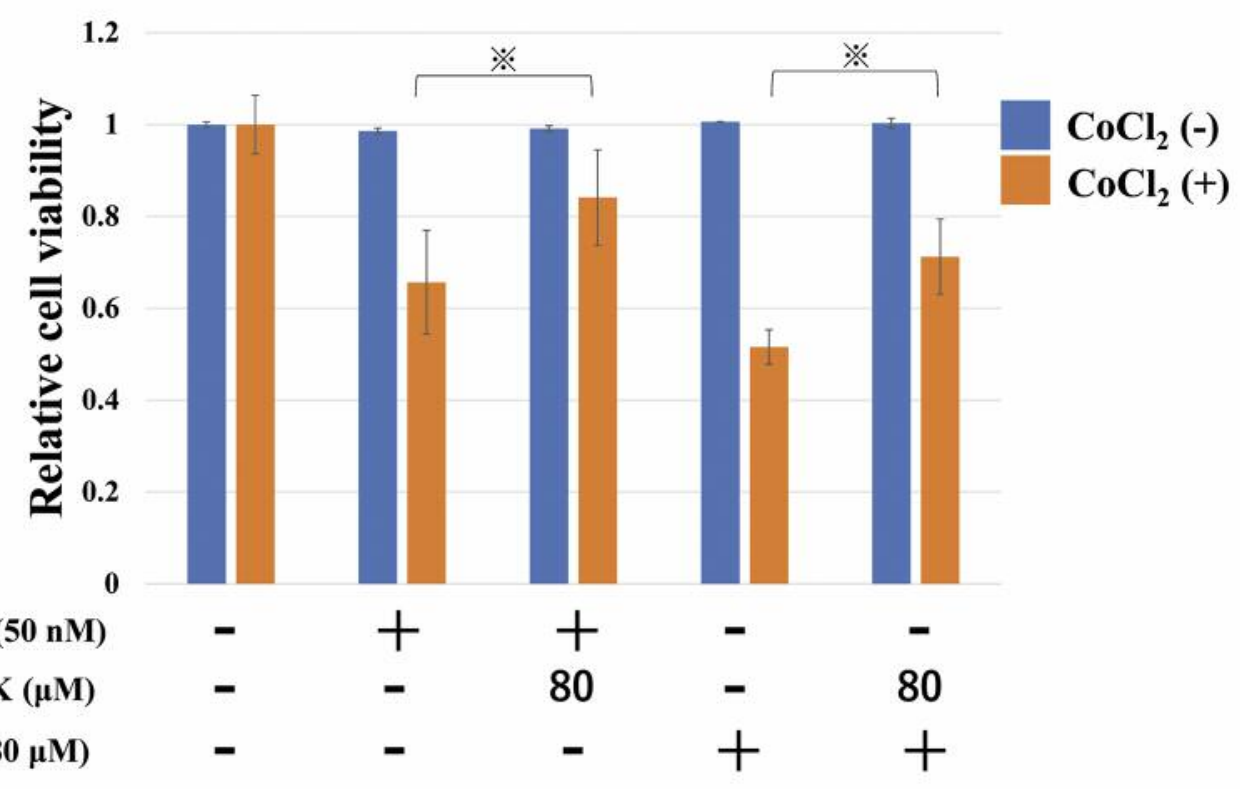

Figure 4. Autophagy induced by hypoxic stress suppresses apoptosis. PANC-1 cells were treated with bafilomycin or LY294002 under either standard culture conditions or $300 \mu \mathrm{M}$ cobalt chloride treatment for $24 \mathrm{~h}$. Expression levels of PARP and actin were determined by western blot analysis (A). Cell viability after $24 \mathrm{~h}$ was assessed using a CCK8 assay. The cell viability of PANC-1 cells treated with autophagy inhibitors with/without Z$V A D(* p<0.05)(B)$.

a clear understanding of how cancer cells adapt to hypoxic and low-nutrient environments, as well as identification of their common mechanism. Even in a nutrient-poor environment, pancreatic cancer cell lines have been reported to display autophagy, which supports cell survival (30). Consistent with the importance of autophagy in the tumor environment, analysis of human surgical specimens has suggested higher autophagy activity in cancer tissues than in non-cancerous tissues (33). Therefore, autophagy is a mechanism that not only enables cells to adapt to hypoxic environments but also supports the survival of the entire microenvironment of pancreatic cancer tissue. Autophagy is thus a favorable therapeutic target.

There are two mechanisms leading to the activation of AMPK (34). The first is a classic mechanism that involves sensing the intracellular AMP/adenosine triphosphate (ATP) ratio. The amount of ATP in cells has been reported to decrease under hypoxic environments (35). The other activation mechanism occurs in response to stimulation by reactive oxygen species (34). Studies carried out in recent years have revealed that reactive oxygen species not only cause DNA and protein damage, but also play a role as intracellular signaling molecules if they are small in number $(36,37)$. There is also a report that reactive oxygen species derived from mitochondria or NADPH oxidase 4 (NOX4) accumulate in cells, which contributes to cell growth or survival, and further enhance cell migration capability under hypoxic conditions $(38,39)$. Detailed analyses should be conducted on the mechanism of AMPK activation caused by hypoxic stress in pancreatic cancer cell lines. 
Autophagy induced under hypoxic conditions is known to have a negative effect on the survival of glioma and breast cancer cell lines in a process described as autophagic cell death (18). However, we revealed for the first time that autophagy induced by hypoxic stress in pancreatic cancer cell lines contributes to cell survival by inhibiting apoptosis and could be useful as a therapeutic target in pancreatic cancer. We have also clarified the importance of the autophagymediated survival system in hepatic cancer cells in order to adapt to hypoxia (data not shown). Cells utilize autophagy as a system to avoid apoptosis via mitophagy, a type of selective autophagy used as a mechanism to maintain the intracellular redox status (13). A more detailed mechanism in pancreatic cancer cell lines needs to be elucidated.

It is important to note that the cytotoxicity caused by inhibition of autophagy was hypoxic environment-selective in our study and only showed a limited effect under normal culture conditions. Reduced glutathione, which has an antioxidant effect, has been reported to decrease under hypoxic environments (40). Therefore, the hypoxic environment-selective cytotoxicity caused by autophagy inhibition likely occurred as a result of impaired antioxidant potential and increased production of reactive oxygen species due to mitophagy failure, which led to excessive accumulation of reactive oxygen species in the cells. Under normal conditions, the antioxidant status could be maintained in comparison the hypoxic environment. In addition, damaged mitochondria requiring mitophagy were small in number under normal conditions. Thus, cytotoxicity was limited, even in autophagy avoidance. A more detailed molecular mechanism focusing on the intracellular redox status should be examined in the future.

In conclusion, this study demonstrated the protective role of autophagy induced by hypoxic stress in pancreatic cancer cells. Blockade of the autophagy pathway could be an attractive therapeutic target in pancreatic cancer.

\section{Conflicts of Interest}

The Authors declare that they have no conflicts of interest.

\section{Acknowledgements}

The Authors thank Makiko Sato and Akiko Sakuyama from the Department of Preventive Medicine, Tokai University School of Medicine, for excellent secretarial support and Kazuhiro Yoshida from the Support Center for Medical Research and Education, Tokai University, for excellent technical support. The Authors would also like to thank Editage (www.editage.jp) for English language editing. This study was supported in part by 2016 Research and Study Project of Tokai University Educational System General Research Organization (SO), 2016 Tokai University School of Medicine Research Aid (SO), 2017 Research and Study Program of Tokai University Educational System General Research Organization (SO), 2017 Tokai University School of Medicine Research Aid (HE) and Grant-in-Aid for Scientific
Research (23701113 and 26870600 to HE) from the Ministry of Education, Culture, Sports, Science, and Technology, Japan.

\section{References}

1 Matsuda T, Ajiki W, Marugame T, Ioka A, Tsukuma H and Sobue T: Population-based survival of cancer patients diagnosed between 1993 and 1999 in Japan: a chronological and international comparative study. Jpn J Clin Oncol 41(1): 40-51, 2010.

2 Vincent A, Herman J, Schulick R, Hruban RH and Goggins M: Pancreatic cancer. The Lancet 378(9791): 607-620, 2011.

3 Reni M, Cordio S, Milandri C, Passoni P, Bonetto E, Oliani C, Luppi G, Nicoletti R, Galli L, Bordonaro R, Passardi A, Zerbi A, Balzano G, Aldrighetti L, Staudacher C, Villa E and Di Carlo V: Gemcitabine versus cisplatin, epirubicin, fluorouracil, and gemcitabine in advanced pancreatic cancer: a randomised controlled multicentre phase III trial. The Lancet Oncology 6(6): 369-376, 2005.

4 Brown JM and Giaccia AJ: The unique physiology of solid tumors: opportunities (and problems) for cancer therapy. Cancer Res 58(7): 1408-1416, 1998.

5 Jain RK: Molecular regulation of vessel maturation. Nat Med 9(6): 685-693, 2003.

6 Thomlinson RH and Gray LH: The histological structure of some human lung cancers and the possible implications for radiotherapy. Br J Cancer 9(4): 539-549, 1955.

7 Less JR, Skalak TC, Sevick EM and Jain RK: Microvascular architecture in a mammary carcinoma: branching patterns and vessel dimensions. Cancer Res 51(1): 265-273, 1991.

8 Liu L, Ning X, Sun L, Zhang H, Shi Y, Guo C, Han S, Liu J, Sun S, Han Z, Wu K and Fan D: Hypoxia-inducible factor- $1 \alpha$ contributes to hypoxia-induced chemoresistance in gastric cancer. Cancer Science 99(1): 121-128, 2008.

9 Song J, Qu Z, Guo X, Zhao Q, Zhao X, Gao L, Kai Sun, Feng Shen, Mengchao $\mathrm{Wu}$ and Lixin Wei: Hypoxia-induced autophagy contributes to the chemoresistance of hepatocellular carcinoma cells. Autophagy 5(8): 1131-1144, 2009.

10 Sullivan R, Pare GC, Frederiksen LJ, Semenza GL and Graham $\mathrm{CH}$ : Hypoxia-induced resistance to anticancer drugs is associated with decreased senescence and requires hypoxiainducible factor-1 activity. Mol Cancer Ther 7(7): 1961-1973, 2008.

11 Yokoi K and Fidler IJ: Hypoxia increases resistance of human pancreatic cancer cells to apoptosis induced by gemcitabine. Clin Cancer Res 10(7): 2299-2306, 2004.

12 Semenza GL: Hypoxia-inducible factor 1: master regulator of O2 homeostasis. Curr Opin Genet Dev 8(5): 588-594, 1998.

13 Zhang H, Bosch-Marce M, Shimoda LA, Tan YS, Baek JH, Wesley JB, Gonzalez FJ and Semenza GL: Mitochondrial autophagy is an HIF-1-dependent adaptive metabolic response to hypoxia. J Biol Chem 283(16): 10892-10903, 2008.

14 Bellot G, Garcia-Medina R, Gounon P, Chiche J, Roux D, Pouyssegur $\mathrm{J}$ and Mazure NM: Hypoxia-induced autophagy is mediated through hypoxia-inducible factor induction of BNIP3 and BNIP3L via their BH3 domains. Mol Cell Biol 29(10): 2570-2581, 2009.

15 Mizushima N, Yamamoto A, Matsui M, Yoshimori $\mathrm{T}$ and Ohsumi Y: In vivo analysis of autophagy in response to nutrient starvation using transgenic mice expressing a fluorescent autophagosome marker. Mol Biol Cell 15(3): 1101-1111, 2004. 
16 Lum JJ, Bauer DE, Kong M, Harris MH, Li C, Lindsten T and Thompson CB: Growth factor regulation of autophagy and cell survival in the absence of apoptosis. Cell 120(2): 237-248, 2005.

17 Bursch W, Ellinger A, Kienzl H, Torok L, Pandey S, Sikorska M, Walker R and Hermann RS: Active cell death induced by the anti-estrogens tamoxifen and ICI 164384 in human mammary carcinoma cells (MCF-7) in culture: the role of autophagy. Carcinogenesis 17(8): 1595-1607, 1996.

18 Azad MB, Chen Y, Henson ES, Cizeau J, McMillan-Ward E, Israels SJ and Gibson SB: Hypoxia induces autophagic cell death in apoptosis-competent cells through a mechanism involving BNIP3. Autophagy 4(2): 195-204, 2008.

19 Zhang R, Zhu F, Ren J, Huang L, Liu P and Wu G: Beclin1/PI3K-mediated autophagy prevents hypoxia-induced apoptosis in EAhy926 cell line. Cancer Biother Radiopharm 26(3): 335-343, 2011.

20 Tasdemir E, Maiuri MC, Galluzzi L, Vitale I, Djavaheri-Mergny M, D'amelio M, Criollo A, Morselli E, Zhu C, Harper F, Nannmark U, Samara C, Pinton P, Vicencio JM, Carnuccio R, Moll UM, Madeo F, Paterlini-Brechot P, Rizzuto R, Szabadkai G, Pierron G, Blomgren K, Tavernarakis N, Codogno P, Cecconi $\mathrm{F}$ and Kroemer G: Regulation of autophagy by cytoplasmic p53. Nat Cell Biol 10(6): 676-687, 2008.

21. Endo H, Niioka M, Kobayashi N, Tanaka M and Watanabe T: Butyrate-producing probiotics reduce nonalcoholic fatty liver disease progression in rats: new insight into the probiotics for the gut-liver axis. PLoS One 8(5): e63388, 2013.

22. Fan RH, Chen PS, Zhao D and Zhang WD: Hypoxia induced by $\mathrm{CoCl} 2$ influencing the expression and the activity of matrix metalloproteinase-2 in rat hepatic stellate cells. Zhonghua Gan Zang Bing Za Zhi 15(9): 654-657, 2007.

23. Karovic O, Tonazzini I, Rebola N, Edström E, Lövdahl C, Fredholm BB and Elisabetta Dare: Toxic effects of cobalt in primary cultures of mouse astrocytes: similarities with hypoxia and role of HIF-1 $\alpha$. Biochem Pharmacol 73(5): 694-708, 2007.

24. Blommaart EF, Luiken JJ, Blommaart PJ, van Woerkom GM and Meijer AJ: Phosphorylation of ribosomal protein S6 is inhibitory for autophagy in isolated rat hepatocytes. J Biol Chem 270(5): 2320-2326, 1995.

25 Zhou Q, Liu H, Sun Q, Zhang L, Lin H, Yuan G, Zhang L and Chen Z: Adenosine Monophosphate-activated Protein Kinase/Mammalian Target of Rapamycin-dependent Autophagy Protects Human Dental Pulp Cells against Hypoxia. J Endod 39(6): 768-773, 2013.

26 Bowman EJ, Siebers A and Altendorf K: Bafilomycins: a class of inhibitors of membrane ATPases from microorganisms, animal cells, and plant cells. Proc Natl Acad Sci USA 85(21): 7972-7976, 1988.

27 Yoshimori T, Yamamoto A, Moriyama Y, Futai M and Tashiro Y: Bafilomycin A1, a specific inhibitor of vacuolar-type H(+)-ATPase, inhibits acidification and protein degradation in lysosomes of cultured cells. J Biol Chem 266(26): 17707-17712, 1991.

28 Blommaart EF, Krause U, Schellens JP, Vreeling-Sindelárová H and Meijer AJ: The phosphatidylinositol 3 kinase inhibitors wortmannin and LY294002 inhibit autophagy in isolated rat hepatocytes. European Journal of Biochemistry 243(1-2): 240-246, 1997.
29 Zhou G, Myers R, Li Y, Chen Y, Shen X, Fenyk-Melody J, Wu M, Ventre J, Doebber T, Fujii N, Musi N Hirshman MF, Goodyear LJ and Moller DE: Role of AMP-activated protein kinase in mechanism of metformin action. J Clin Invest 108(8): 1167-1174, 2001.

30 Kim SE, Park H, Jeong HK, Kim M, Kim M, Bae O and Baek $\mathrm{SH}$ : Autophagy sustains the survival of human pancreatic cancer PANC-1 cells under extreme nutrient deprivation conditions. Biochem Biophys Res Commun 463(3): 205-210, 2015.

$31 \mathrm{Hu}$ Y, Yin Y, Liu H, Feng Y, Bian Z, Zhou L, Zhang JW, Fei BJ, Wang YG and Huang ZH: Glucose deprivation induces chemoresistance in colorectal cancer cells by increasing ATF4 expression. World Journal of Gastroenterology 22(27): 6235$6245,2016$.

32 Yakisich JS, Venkatadri R, Azad $\mathrm{N}$ and Iyer AKV: Chemoresistance of lung and breast cancer cells growing under prolonged periods of serum starvation. J Cell Physiol 232(8): 2033-2043, 2017.

33 Hirayama A, Kami K, Sugimoto M, Sugawara M, Toki N, Onozuka H, Kinoshita T, Saito N, Ochiai A, Tomita M, Esumi H and Soga T: Quantitative metabolome profiling of colon and stomach cancer microenvironment by capillary electrophoresis time-of-flight mass spectrometry. Cancer Res 69(11): 4918-4925, 2009.

34 Hardie DG, Ross FA and Hawley SA: AMPK: a nutrient and energy sensor that maintains energy homeostasis. Nature Reviews Molecular Cell Biology 13(4): 251-262, 2012.

35 Heerlein K, Schulze A, Hotz L, Bartsch P and Mairbaurl H: Hypoxia decreases cellular ATP demand and inhibits mitochondrial respiration of a549 cells. American Journal of Respiratory Cell and Molecular Biology 32(1): 44-51, 2005.

36 Owada S, Shimoda Y, Tsuchihara K and Esumi H: Critical role of $\mathrm{H}_{2} \mathrm{O}_{2}$ generated by NOX4 during cellular response under glucose deprivation. PLoS One 8(3): e56628, 2013.

37 Nogueira V and Hay N: Molecular pathways: reactive oxygen species homeostasis in cancer cells and implications for cancer therapy. Clin Cancer Res 19(16): 4309-4314, 2013.

38 Chandel NS, McClintock DS, Feliciano CE, Wood TM, Melendez JA, Rodriguez AM and Schumacker PT: Reactive oxygen species generated at mitochondrial complex III stabilize hypoxia-inducible factor-1alpha during hypoxia: a mechanism of O2 sensing. J Biol Chem 275(33): 25130-25138, 2000.

39 Diebold I, Petry A, Hess J and Gorlach A: The NADPH oxidase subunit NOX4 is a new target gene of the hypoxia-inducible factor-1. Mol Biol Cell 21(12): 2087-2096, 2010.

40 Mansfield KD, Simon MC and Keith B: Hypoxic reduction in cellular glutathione levels requires mitochondrial reactive oxygen species. J Appl Physiol 97(4): 1358-1366, 2004.

Received July 5, 2017

Revised July 20, 2017

Accepted July 21, 2017 